\title{
A RECIPROCITY THEOREM FOR ERGODIC ACTIONS
}

\author{
BY \\ KENNETH LANGE
}

\begin{abstract}
An analogue of the Frobenius Reciprocity Theorem is proved for virtual groups over a locally compact separable group $G$. Specifically, an ergodic analytic Borel $G$-space $M(V \pi)$ is constructed from a virtual group $V$ and a homomorphism $\pi: V \rightarrow G$ of $V$ into $G$. This construction proves to be functorial for the category of virtual groups over $G$; in fact, it is a left adjoint of the functor which takes an ergodic analytic Borel $G$-space $T$ into the virtual group $T \times G$ together with projection $\rho: T \times G \rightarrow G$ onto $G$. Examples such as Kakutani's induced transformation and flows under functions show the scope of this construction.

A method for constructing the product of two virtual groups is also presented. Some of the structural properties of the product virtual group are deduced from those of the components. Finally, for virtual groups $\pi_{1}: V_{1} \rightarrow G_{1}$ and $\pi_{2}: V_{2} \rightarrow G_{2}$ over groups $G_{1}$ and $G_{2}$ respectively, the adjoint functor construction applied to $\pi_{1} \times \pi_{2}: V_{1} \times V_{2} \rightarrow G_{1} \times G_{2}$ is shown to give the product of the $G_{1}$-space derived from $\pi_{1}: V_{1} \rightarrow G_{1}$ and the $G_{2}$-space derived from $\pi_{2}: V_{2} \rightarrow G_{2}$, up to suitably defined isomorphism.
\end{abstract}

I. Introduction. In this brief introduction, we shall try to put into its proper historical perspective the main result proved in this paper. The present work can be viewed as the confluence of two different trends in modern functional analysis. The first is classical ergodic theory, whose main aim can still be considered to be that of classifying measure preserving transformations by extending the methods of spectral theory. It is well known that the classification of measure preserving transformations with discrete spectra is the only classification that has been completely achieved by spectral methods in Hilbert space. For more general measure preserving transformations, the sole innovation since the study began with von Neumann in the early thirties has been the introduction of the notion of entropy. This notion has been moderately successful in giving a new invariant of measure preserving transformations which can distinguish several conjugacy classes, but the reason why it works remains unclear. Furthermore, aside from entropy, no new invariant for the conjugacy problem of measure preserving transformations has come to the surface. It seems rather that the problem of conjugacy of measure preserving transformations will turn out to be as difficult as the problem of conjugacy of arbitrary bounded operators in Hilbert space.

The second trend is the theory of group representations, which has been far more successful than ergodic theory ever since the methods of functional analysis

Received by the editors May 3, 1971.

AMS 1970 subject classifications. Primary 22D40, 28A65; Secondary 22D30, 28 A60.

Key words and phrases. Transformation space, analytic Borel space, ergodic, groupoid, virtual group, adjoint functor, flow under a function.

Copyright (C) 1972, American Mathematical Society 
were first applied to it by Weil, Segal, Mackey and others. More recently, several notions of the theory of group representations have been considerably streamlined by the methods of categorical algebra. See for a survey the paper of Lawvere [4].

It was Mackey who first brought to the attention of the mathematical public the possible analogies between group representations and group actions, and the possibility of carrying over to the study of group actions-in particular, those associated with measure preserving transformations-various of the notions formerly used only for linear representations. The work begun by Mackey, in a series of rather cryptic notes, has only recently been expanded and given a coherent form in the work of Ramsay. By ingenious ad hoc methods, Mackey was able to show that several of the "constructions" classically used in measure theory could be greatly extended by using analogous notions from the theory of group representations. Nevertheless, in Mackey's budding theory these "constructions" remained isolated coincidences.

It is our purpose to show that at least one of Mackey's constructions, perhaps the most important one, can be greatly extended and given a complete explanation within the context of categorical algebra. In fact, we will go one step further than Mackey, and show that his construction can be strengthened to give an analogue of the Frobenius Reciprocity Theorem for group actions, namely actions of groups on measurable spaces as defined below.

It has been known for some time that the Frobenius Reciprocity Theorem is a particular instance of the construction of the adjoint of a certain functor, namely the restriction functor of a representation. We take this as our background, and we show that with suitable definitions and constructions one can similarly construct the adjoint functor of an ergodic group action. We have been fortunate enough to carry through the complete formulation of this idea, despite a large number of measure theoretic details, which at present remain quite extensive, but which we hope that later workers in the field will be able to whittle down.

We give several applications of our construction to show that it is indeed an extension of the Reciprocity Theorem. Perhaps the most enlightening application is the one that gives us the construction of a flow under a function.

ACKNOWLEDGEMENTS. Some of the results of this paper were announced in [15]. The body of the paper is taken from the author's Ph.D. thesis at M.I.T. He wishes to thank his thesis advisor, Professor Gian-Carlo Rota, for suggesting the main theorem and Professor Arlan Ramsay for help in reaching the final formulation of that theorem. Note that Professor Ramsay's paper [13] partially overlaps the present work.

II. Virtual groups and their functorial properties. Recall that a group is a small category with one object, where every morphism has an inverse. A groupoid is a small category where every morphism has an inverse. In a groupoid $V$ the set of objects can be identified with the unit morphisms. For every morphism $f \in V$ denote the inverse of $f$ by $f^{-1}$. Then $f f^{-1}$ is the left unit of $f$ and $f^{-1} f$ is the right unit of $f$. 
We refer to Moore [9, pp. 2-18] for the definitions of the measure theoretic and Borel space terms appearing hereafter. An analytic Borel groupoid is a groupoid $V$, together with an analytic Borel space structure on the set of morphisms, and a measure class $C$ such that: (a) The domain $D$ of composition of two morphisms is a Borel subset of $V \times V$, under the product Borel structure. (b) Composition $(f, h) \rightarrow f h$ and inversion $f \rightarrow f^{-1}$ are Borel maps from $D$ to $V$ and $V$ to $V$ respectively. (c) Inversion leaves invariant the measure class $C$, i.e. takes null sets into null sets.

Condition (d) must be prefaced by a few explanatory remarks. Let $U$ denote the collection of units of $V$. Then $U$ is a Borel subset of $V$ and hence forms an analytic Borel space under its relative Borel structure. (See Chapter 4 of [13].) The right unit map $d: V \rightarrow U$ and the left unit map $r: V \rightarrow U$ are both Borel. $d: V \rightarrow U$ induces a measure class $\bar{C}$ on $U$, setting $\bar{\mu}(A)=\mu\left(d^{-1}(A)\right)$ for $\mu$ in $C$. Any $\mu$ in $C$ satisfies $\mu=\int \mu_{s} d \bar{\mu}(s)$, where $\mu_{s}$ is a measure on $V$ living on $d^{-1}(s)$, and for a fixed Borel set $A$ in $V$, the function $s \rightarrow \mu_{s}(A)$ is Borel on $U$. The fibering is unique: if $\mu=\int \nu_{s} d \bar{\mu}(s)$, then $\nu_{s}=\mu_{s}$ for $\bar{C}$-almost all $s$; furthermore, changing $\mu$ to an equivalent measure does not change the measure class of $\mu_{s}$ for $\bar{C}$-almost all $s$; this leads to measure classes $C_{s}$ on almost all fibers $d^{-1}(s)$. (d) For $s \in U$ and $r(f)=s$ the map $h \rightarrow h f$ carries $d^{-1}(s)$ bijectively to $d^{-1}(d(f))$ and $C_{s}$ to $C_{s} f$. We require that $C_{r(f)} f=C_{d(f)}$ for all $f$ with $r(f)$ and $d(f)$ in some conull (complement of a null) Borel set $U_{0}$ of $U$.

Before defining virtual group we call attention to the fact that the measure class $\bar{C}$ on $U$ can be equally well defined by $r: V \rightarrow U$, setting $\bar{\mu}(A)=\mu\left(r^{-1}(A)\right)$ for $\mu$ in $C$. This follows from the invariance of $C$ under inversion. Moreover, each $\mu$ in $C$ has a fiber measure decomposition with respect to $r: V \rightarrow U$; this leads to measure classes on almost all the fibers $r^{-1}(s)$ and a condition equivalent to (d) concerning the invariance of the fiber measure classes with respect to transformations $h \rightarrow f h$.

An analytic Borel groupoid $(V, C)$ is said to be ergodic whenever every real Borel function $\phi$ on the sets of units $U$ such that $\phi(d(f))=\phi(r(f))$ for almost all $f$ in $V$ is $\bar{C}$-almost everywhere constant. For short, an ergodic analytic Borel groupoid is called a virtual group.

In a virtual group $(V, C)$ let $U_{0}$ be a $\bar{C}$-conull Borel subset of $U$. Taking all $f \in V$ such that both $d(f)$ and $r(f)$ are in $U_{0}$, we obtain another virtual group, the inessential contraction (abbreviated i.c.) $V \mid U_{0}$. Again, say that two units $u$ and $v$ are equivalent when $d(f)=u$ and $r(f)=v$ for some $f$ in $V$; if $A \subset U$, write [A] for the saturation of $A$ (set of units equivalent to some unit of $A$ ) under this equivalence relation; note that $[A]=r\left(d^{-1}(A)\right)$ and that $[A]$ is analytic if $A$ is a Borel set.

An analytic Borel groupoid $(V, C)$ is termed essentially transitive whenever there is a unit $u$ in $U$ whose saturation $[u]$ is conull in $U$. An essentially transitive analytic Borel groupoid is ergodic, but not conversely. $(V, C)$ is termed essentially principal whenever there is a conull set of units $U_{0}$ such that for every $u$ in $U_{0}$, $\{f \in V: d(f)=r(f)=u\}=\{u\}$.

A strict homomorphism $\psi$ between virtual groups $\left(V_{1}, C_{1}\right)$ and $\left(V_{2}, C_{2}\right)$ is a functor 
from $V_{1}$ to $V_{2}$ which is also a Borel map, and such that if $\bar{\psi}$ is the associated map of the units $U_{1}$ of $V_{1}$ to the units $U_{2}$ of $V_{2}$, then $\bar{\psi}^{-1}(A)$ is a $\bar{C}_{1}$-null set for every saturated $\bar{C}_{2}$-null set $A$. (This apparently different definition is equivalent to Ramsay's. See Lemma 6.6 of [13].) A homomorphism of $\left(V_{1}, C_{1}\right)$ to $\left(V_{2}, C_{2}\right)$ is a Borel map whose restriction to some i.c. is a strict homomorphism. Two homomorphisms $\psi_{1}$ and $\psi_{2}:\left(V_{1}, C_{1}\right) \rightarrow\left(V_{2}, C_{2}\right)$ are strictly similar if $\theta\left(r_{1}(f)\right) \psi_{1}(f)=\psi_{2}(f) \phi\left(d_{1}(f)\right)$ for all $f \in V_{1}$ and for some Borel map $\theta: U_{1} \rightarrow V_{2}$ for which both sides are defined, where $U_{1}, d_{1}, r_{1}$ refer to the units and unit maps of $V_{1} . \psi_{1}$ and $\psi_{2}:\left(V_{1}, C_{1}\right) \rightarrow\left(V_{2}, C_{2}\right)$ are similar if there is an i.c. of $V_{1}$ on which they are strictly similar. Similarity is an equivalence relation. Given homomorphisms $\psi: V_{1} \rightarrow V_{2}$ and $\kappa: V_{2} \rightarrow V_{3}$, the composition $\kappa \circ \psi$ may not be a homomorphism. However there is a homomorphism $\phi: V_{1} \rightarrow V_{2}$ similar to $\psi$ such that $\kappa \circ \phi$ is also a homomorphism. ( $\kappa$ and $\phi$ are said to be composable.) Similarity classes $[\psi]$ of homomorphisms $\psi$ are preserved under composition. (See Chapter 6 of [13] for a discussion of these delicate points.) Taking virtual groups as objects and similarity classes of homomorphisms as morphisms, one obtains a category. Two virtual groups $V_{1}$ and $V_{2}$ which are isomorphic in this category are called similar. In other words, there are morphisms $[\kappa]: V_{1} \rightarrow V_{2}$ and $[\psi]: V_{2} \rightarrow V_{1}$ such that $[\psi] \circ[\kappa]=\left[\mathrm{id}_{1}\right]$ and $[\kappa] \circ[\psi]=\left[\mathrm{id}_{2}\right]$, where $\mathrm{id}_{1}$ and $\mathrm{id}_{2}$ are the identity maps on $V_{1}$ and $V_{2}$ respectively. Similarity of virtual groups is an equivalence relation.

A locally compact separable group $G$ is a virtual group when endowed with its Haar measure class. A virtual group $V$, together with a homomorphism $\pi: V \rightarrow G$, briefly $V \pi$, will be called a virtual group over $G$. The Mackey category $\mathrm{M}(G)$ of $G$ has the virtual groups over $G$ as objects, and as morphisms the similarity classes of homomorphisms which make the obvious triangle over $G$ commute, namely, which commute with the action of $[\pi]$.

The category $\mathrm{R}(G)$ of ergodic actions of $G$ has as objects the transformation spaces $T$ of $G$, namely: (a) $T$ is an analytic Borel space; (b) the map $(t, x) \rightarrow t x$ of $T \times G \rightarrow T$ is Borel; (c) $T$ has a measure class $C$ which is invariant under the set of Borel automorphisms $t \rightarrow t x$; (d) $(T, C)$ is ergodic: the only invariant Borel sets are null or conull. The morphisms of $\mathrm{R}(G)$ are equivalence classes of maps, as follows: $T_{1}$ and $T_{2}$ being objects, consider all Borel maps $\phi: T_{1} \rightarrow T_{2}$ such that: (a) there is a conull invariant analytic subset of $T_{1}$ on which $\phi$ is $G$-equivariant, i.e. $\phi(t x)=\phi(t) x ;$ (b) if $N$ is a null invariant set in $T_{2} ; \phi^{-1}(N)$ is null too. Two maps $\phi$ and $\psi$ are equivalent if there is a Borel map $\alpha: T_{1} \rightarrow G$ such that $\phi(t) \alpha(t)=\psi(t)$ and $\alpha(t x)=x^{-1} \alpha(t) x$ for all $t$ in some conull invariant analytic subset of $T_{1}$. This equivalence is preserved under composition of maps; the equivalence classes $[\phi]$ are the morphisms of $\mathrm{R}(G)$. Note here and for future reference that the collection of analytic sets is closed under intersection and the formation of images and inverse images under Borel maps between analytic spaces.

Before starting the proof of our main theorem, we must expand on Mackey's method of turning an ergodic $G$-space into a virtual group over $G$ [5], [6]. The 
kernel of the idea is as follows: For a given ergodic action of $G$ on $T$, give $T \times G$ the product Borel structure and product measure class and define $\pi: T \times G \rightarrow G$ as projection onto $G$. Defining $(s, x)(t, y)=(s, x y)$ whenever $s x=t$ gives $T \times G$ a groupoid structure whose units can be naturally identified with the set $T$. (The set of units is really $T \times\{e\}$, where $e$ is the identity of $G$.) This construction maps the objects of $\mathrm{R}(G)$ into the objects of the Mackey category $\mathrm{M}(G)$.

THEOREM 1. There is a functor $R: \mathrm{R}(G) \rightarrow \mathrm{M}(G)$ extending the above construction, which is faithful on objects and morphisms and whose image is a full subcategory of the Mackey category.

Proof. Given a morphism class $[\phi]: T_{1} \rightarrow T_{2}$ in $\mathrm{R}(G)$, let us define a similarity class of homomorphisms $R([\phi]): R\left(T_{1}\right) \rightarrow R\left(T_{2}\right)$ in $\mathrm{M}(G)$. First observe that the Borel map $(t, x) \rightarrow(\phi(t), x)=R(\phi)(t, x)$ is a homomorphism between $R\left(T_{1}\right)$ and $R\left(T_{2}\right)$. Now if $\alpha: T_{1} \rightarrow G$ provides an equivalence between the two $\mathrm{R}(G)$ maps $\phi$ and $\psi$, then $(\phi(t), x)(\phi(t x), \alpha(t x))=(\phi(t), \alpha(t))(\psi(t), x)$ on some i.c. of $R\left(T_{1}\right)$, and so $t \rightarrow(\phi(t), \alpha(t))$ implements a similarity between $R(\phi)$ and $R(\psi)$. Thus $[R(\phi)]$ $=R([\phi])$ gives a meaningful definition. It is relatively simple to check that $R$ preserves the composition of morphisms.

$R$ is clearly faithful on objects. To demonstrate that $R$ is faithful on morphisms, it suffices to prove that if $\phi, \psi: T_{1} \rightarrow T_{2}$ are $\mathrm{R}(G)$ maps such that $R(\phi)$ and $R(\psi)$ are similar, then $\phi$ and $\psi$ are equivalent. With this in mind note that $(\phi(t), x)(\theta(t x), \beta(t x))=(\theta(t), \beta(t))(\psi(t), x)$ on some i.c., where $t \rightarrow \theta(t)$ and $t \rightarrow \beta(t)$ are Borel maps. Obviously $\phi(t)=\theta(t)$ here, so that $\phi(t) \beta(t)=\psi(t)$. Furthermore, $\beta(t x)=x^{-1} \beta(t) x$ on this i.c. Considering $G$ to be a $G$-space with action defined by $y \cdot x=x^{-1} y x$, one can replace $\beta$ by a Borel map $\alpha: T_{1} \rightarrow G$ which agrees with $\beta$ except on a null set and which is $G$-equivariant on an invariant analytic conull set of $T_{1}$. (Use the proof of Theorem 3.6 of [13].) Thus there is a common invariant analytic conull set $A$ where $\phi, \psi$ and $\alpha$ are all $G$-equivariant. Since $\phi(t) \alpha(t)=\psi(t)$ almost everywhere, one can reduce $A$ to a set $B$ with the same properties such that $\phi(t) \alpha(t)=\psi(t)$ for all $t \in B$.

To show that $R$ maps onto a full subcategory assume $\kappa: R\left(T_{1}\right) \rightarrow R\left(T_{2}\right)$ is a $\mathrm{M}(G)$ homomorphism. Let $\pi_{i}(i=1,2)$ be the projection of $R\left(T_{i}\right)$ onto $G$. Then there is a Borel map $\theta: T_{1} \rightarrow G$ and an i.c. of $R\left(T_{1}\right)$ on which $\kappa$ is strict and $\pi_{2} \circ \kappa(t, x) \theta(t x)=\theta(t) \pi_{1}(t, x)$. Put $\kappa(t, x)=(\sigma(t, x), \delta(t, x))$ and look at $\kappa((t, e)(t, x))$. It follows first that $\sigma(t, x)=\sigma(t, e)$ on the i.c. and second that the Borel map $(t, x)$ $\rightarrow(\sigma(t, e), \delta(t, x))$ is a homomorphism agreeing with $\kappa$ there. Define a homomorphism $\psi$ similar to $\kappa$ by

$$
(t, x) \rightarrow\left(\sigma(t, e) \theta(t), \theta(t)^{-1}\right) \kappa(t, x)\left(\sigma(t x, e) \theta(t x), \theta(t x)^{-1}\right)^{-1}=\psi(t, x)
$$

on the i.c. and constant elsewhere. Then $\pi_{2} \circ \psi(t, x)=\pi_{1}(t, x)=x$ and a glance at $\psi\left((t, x)\left(t x, x^{-1}\right)\right)$ reveals that $\bar{\psi}(t)=\sigma(t, e) \theta(t)$ satisfies $\sigma(t, e) \theta(t) x=\sigma(t x, e) \theta(t x)$ on the above i.c. By the proof of Theorem 3.6 of [13] there is a Borel map $\eta: T_{1} \rightarrow T_{2}$ 
which agrees with $t \rightarrow \sigma(t, e) \theta(t)$ except on a null set and is $G$-equivariant on an invariant analytic conull set. Thus $R(\eta)$ agrees with $\psi$ on an i.c. and so is similar to $\kappa$. This completes the proof of the theorem.

\section{The Adjoint Functor Theorem: Statement and proof.}

\section{THEOREM 2. The functor $R$ has a left adjoint $M$.}

The proof is long and will be broken into a series of propositions. $M$ will also be called the Mackey Functor.

Proposition 2.1. Given a virtual group $V \pi$ over $G$ one can construct an ergodic analytic Borel $G$-space $M(V \pi)$.

Proof. By passing to an i.c. if necessary, one can assume that $\pi$ is a strict homomorphism. For convenience choose a specific finite measure $v$ in the measure class of $V . \bar{\nu}$ will denote the measure induced on the units $U$ of $V$ by the right unit map $d$.

The product space $U \times G$ is an analytic Borel $G$-space under the $G$-action $(u, x) y=\left(u, y^{-1} x\right)$. Let $\mu$ be a finite measure in the Haar measure class of $G$. Then the product measure $\bar{\nu} \times \mu$ is quasi-invariant under the action of $G$. To see this apply Fubini's Theorem and the quasi-invariance of $\mu$. Next define an equivalence relation $\sim$ on $U \times G$ which commutes with the action of $G .(u, x) \sim(w, y)$ iff $(r(f), x)=(u, x)$ and $(d(f), x \pi(f))=(w, y)$ for some $f \in V$. Call a set $A$ in the collection of equivalence classes $U \times G / \sim$ measurable iff $A$ (when viewed as a set in $U \times G)$ differs from a Borel set in $U \times G$ by a null set. The family of measurable sets of $U \times G / \sim$ forms a $\sigma$-algebra. Furthermore, $\bar{\nu} \times \mu$ induces a measure class on the measurable sets of $U \times G / \sim$ which is complete, i.e. every set which is contained in a null set of $U \times G / \sim$ is itself measurable. From here on $U \times G / \sim$ will be considered with the Borel structure of measurable sets and the induced measure class.

Next note that the measurable sets in $U \times G / \sim$ give rise to a $G$-invariant closed subalgebra $M A(U \times G / \sim)$ of the measure algebra $M A(U \times G)$. Equivalently, $M A(U \times G / \sim)$ is the closed subalgebra of $M A(U \times G)$ generated by the measurable sets in $U \times G$ which are saturated with respect to $\sim$. Since every closed subalgebra of a standard measure algebra is standard, a theorem of Mackey, given as Theorem 3.3 of [13], implies the existence of a standard Borel $G$-space $M(V \pi)$ equipped with an invariant measure class such that its associated measure algebra is isomorphic as a Boolean $G$-space to $M A(U \times G / \sim) . M(V \pi)$ is the standard (hence necessarily analytic) Borel $G$-space we are seeking.

It remains to demonstrate that the action of $G$ on $M(V \pi)$ is ergodic. Clearly it is enough to show that the action of $G$ on $M A(U \times G / \sim)$ is ergodic. (See [9] for a discussion of the equivalent notions of ergodicity on analytic Borel $G$-spaces.) Thus let $D$ be a measurable set in $U \times G$ which is the union of $\sim$ equivalence classes and satisfies $\bar{\nu} \times \mu(D \triangle D x)=0$ for all $x \in G$, where " $\triangle$ " denotes symmetric difference. Since $U \times G$ is an analytic Borel $G$-space, there is a Borel set $F$ with 
$\bar{\nu} \times \mu(F \triangle D)=0$ and $F=F x$ for $x \in G$. It is not too difficult to see that $F$ must be of the form $B \times G$, where $B$ is a Borel set in $U$. Compute as follows:

$$
\begin{aligned}
0 & =\iint\left|\chi_{B \times G}(u, x)-\chi_{D}(u, x)\right| d \bar{\nu}(u) d \mu(x) \\
& =\iint\left|\chi_{B \times G}(d(f), x)-\chi_{D}(d(f), x)\right| d \nu(f) d \mu(x) \\
& =\iint\left|\chi_{d^{-1}(B)}(f)-\chi_{D}(d(f), x)\right| d \nu(f) d \mu(x) \\
& =\iint\left|\chi_{d^{-1}(B)}(f)-\chi_{D}\left(r(f), x \pi(f)^{-1}\right)\right| d \nu(f) d \mu(x),
\end{aligned}
$$

where the last equality is a consequence of the fact that $D$ is a union of $\sim$ equivalence classes. Applying Fubini's Theorem and the quasi-invariance of $\mu$ to the last equation above gives

$$
0=\iint\left|\chi_{d^{-1}{ }_{(B)}}(f)-\chi_{D}(r(f), x)\right| d \nu(f) d \mu(x) .
$$

Similarly, the quasi-invariance of $\nu$ under $f \rightarrow f^{-1}$ and Fubini's Theorem imply

$$
\begin{aligned}
0 & =\iint\left|\chi_{d^{-1}(B)}\left(f^{-1}\right)-\chi_{D}\left(d\left(f^{-1}\right), x\right)\right| d \nu(f) d \mu(x) \\
& =\iint\left|\chi_{r^{-1}(B)}(f)-\chi_{D}(r(f), x)\right| d \nu(f) d \mu(x) .
\end{aligned}
$$

Adding (*) and $(* *)$ yields

$$
0=\iint\left|\chi_{r^{-1}(B)}(f)-\chi_{d^{-1}(B)}(f)\right| d \nu(f) d \mu(x) .
$$

Hence for some $x, \chi_{B}(d(f))=\chi_{B}(r(f))$ for almost all $f$. Because $V$ is ergodic $B$ must be either null or conull. It follows that $F$ and $D$ are null or conull.

Remarks. Suppose $V \mid U_{0}$ is an i.c. of $V$ and $\sim_{0}$ is the restriction of the equivalence $\sim$ to $U_{0} \times G$. Then the closed subalgebra of $M A(U \times G)$ derived from measurable sets in $U_{0} \times G / \sim_{0}$ is the same as the one derived from $U \times G / \sim$. Hence $M(V \pi)$ and $M\left(V \mid U_{0} \pi_{0}\right)$ are the same, where $\pi_{0}$ is the restriction $\left.\pi\right|_{V \mid U_{0}}$.

If $V \pi$ is in $\mathrm{M}(G)$, then the equivalence relation $\sim$ on $U \times G$ is analytic. This means that as a subset of $(U \times G) \times(U \times G), \sim$ is analytic. Indeed, $\sim$ can be identified with the image of the analytic space $V \times G$ under the Borel map $(f, x)$ $\rightarrow(r(f), x) \times(d(f), x \pi(f))$. Now consider $U \times G / \sim$ with the quotient Borel structure, instead of the Borel structure derived from the $\sim$ saturated measurable sets in $U \times G$. If by chance $U \times G / \sim$ is countably separated, then $U \times G / \sim$ is actually an analytic Borel $G$-space. (See Propositions 2.9 and 3.1 of Chapter 1 of [9].) When $U \times G / \sim$ is an analytic Borel $G$-space, Theorem 7.7 of [13] implies that up to invariant null sets, $U \times G / \sim$ and $M(V \pi)$ are isomorphic as analytic Borel $G$-spaces 
with invariant measure classes. Another sufficient condition for $U \times G / \sim$ to be analytic is the existence of an analytic subset of $U \times G$ meeting each $\sim$ equivalence class in exactly one point. (See Proposition 2.12 of Chapter 1 of [9].)

Let us also observe that for $T$ in $\mathrm{R}(G), M(R(T))$ can be taken to be $T$. In fact, the equivalence relation $\sim$ on $T \times G$ reduces to $(t, x) \sim(t y, x y)$ and so the map $(t, x) \rightarrow t x^{-1}$ factors to give a $G$-equivariant Borel bijection of $T \times G / \sim$ (with the quotient Borel structure) onto $T$. Since $T$ is countably separated, it is possible to prove $T \times G / \sim$ is too. By the above remark $T \times G / \sim$ is an analytic Borel $G$-space. Hence the Borel bijection is even a Borel isomorphism. (See Proposition 2.5 of Chapter 1 of [9].) Finally, it follows from Fubini's Theorem that the measure class induced on $T$ by $(t, x) \rightarrow t x^{-1}$ is the same as the given measure class on $T$.

Note also that we are using a strong form of the axiom of choice in defining $M(V \pi)$. Indeed, there may be many analytic Borel $G$-spaces with measure algebras which are $\sigma$-isomorphic as Boolean $G$-spaces to $M A(U \times G / \sim)$. We select precisely one such space and one $G$-equivariant $\sigma$-isomorphism between its measure algebra and $M A(U \times G / \sim)$. (See [11] for a discussion of this strong form of the axiom of choice.)

Proposition 2.2. Given the morphism $[\kappa]: V_{1} \pi_{1} \rightarrow V_{2} \pi_{2}$ in $\mathrm{M}(G)$, one can construct a $\mathrm{R}(G)$ morphism $M([\kappa]): M\left(V_{1} \pi_{1}\right) \rightarrow M\left(V_{2} \pi_{2}\right)$.

The proof will be given as a sequence of three lemmas. In what follows the sets of units, the unit maps, and the $\sim$ equivalence relations for $V_{1} \pi_{1}$ and $V_{2} \pi_{2}$ will be distinguished by numerical subscripts, e.g., $U_{1}$ will denote the set of units of $V_{1}$. Those homomorphisms in $[\kappa]$ which are not composable with $\pi_{2}$ will be disregarded. This, along with the first remark above, allows us to take $\pi_{1}, \pi_{2}$, and $\kappa$ strict.

LEMMA 2.3. If $\theta\left(r_{1}(f)\right) \pi_{2} \circ \kappa(f)=\pi_{1}(f) \theta\left(d_{1}(f)\right)$ holds on some i.c. of $V_{1}$, then there corresponds a homomorphism $M\left(\kappa_{\theta}\right): M\left(V_{1} \pi_{1}\right) \rightarrow M\left(V_{2} \pi_{2}\right)$.

Proof. Passing to an i.c. we can assume $\pi_{2} \circ \kappa$ strictly similar to $\pi_{1}$ via $\theta$. Consider the Borel map $(u, x) \rightarrow(\bar{\kappa}(u), x \theta(u))=\alpha(u, x)$ of $U_{1} \times G$ to $U_{2} \times G . \alpha$ is $G$-equivariant and factors through the equivalence relations to give a map $U_{1} \times G / \sim_{1} \rightarrow U_{2} \times G / \sim_{2}$. Indeed,

$$
\begin{aligned}
\left(r_{1}(f), x\right) \rightarrow\left(\bar{\kappa}\left(r_{1}(f)\right)\right. & \left., x \theta\left(r_{1}(f)\right)\right) \\
= & \left(r_{2}(\kappa(f)), x \theta\left(r_{1}(f)\right)\right) \sim_{2}\left(d_{2}(\kappa(f)), x \theta\left(r_{1}(f)\right) \pi_{2} \circ \kappa(f)\right) \\
= & \left(\bar{\kappa}\left(d_{1}(f)\right), x \pi_{1}(f) \theta\left(d_{1}(f)\right)\right)
\end{aligned}
$$

and

$$
\left(d_{1}(f), x \pi_{1}(f)\right) \rightarrow\left(\bar{\kappa}\left(d_{1}(f)\right), x \pi_{1}(f) \theta\left(d_{1}(f)\right)\right) .
$$

To show that the factored map induces a $G$-equivariant $\sigma$-homomorphism $M A\left(U_{2} \times G / \sim_{2}\right) \rightarrow M A\left(U_{1} \times G / \sim_{1}\right)$ it is necessary to prove that the inverse image 
of a null set $A$ in $U_{2} \times G / \sim_{2}$ is null. It is also necessary to show that the inverse image of a measurable set is measurable. Consider the first condition. Viewing $A$ as a set in $U_{2} \times G,\left\{u \in U_{2}: A^{u}\right.$ is not null $\}$ is clearly null. Here $A^{u}$ means the section $\{x \in G:(u, x) \in A\}$. Also since $A^{d_{2}(f)}=A^{r_{2}(f)} \pi_{2}(f),\left\{u: A^{u}\right.$ is not null $\}$ is saturated in $U_{2}$. Because $\kappa$ is a homomorphism, $\bar{\kappa}^{-1}\left\{u: A^{u}\right.$ is not null $\}$ is null. Now $\left\{u \in U_{1}: \alpha^{-1}(A)^{u}\right.$ is not null $\}=\bar{\kappa}^{-1}\left\{u \in U_{2}: A^{u}\right.$ is not null $\}$ because $\alpha^{-1}(A)^{u}$ $=\{x \in G:(\bar{\kappa}(u), x \theta(u)) \in A\}=A^{\kappa(u)} \theta(u)^{-1}$. Finally Fubini's Theorem yields that $\alpha^{-1}(A)$ is null.

As for the second condition let $B$ be a measurable set in $U_{2} \times G$ which is saturated with respect to $\sim_{2}$. Then there is a $\sim_{2}$-saturated set $A$ in $U_{2} \times G$ which is analytic and which differs from $B$ by a null set. (See the first part of the proof of Theorem 7.11 of [13].) The inverse image of $A$ is analytic in $U_{1} \times G$ and hence measurable. Furthermore, the inverse images of $A \backslash B$ and $B \backslash A$ are null and thus also measurable. If we write $B$ as $(A \backslash(A \backslash B)) \cup(B \backslash A)$, it follows that the inverse image of $B$ is measurable too.

To complete the proof of the lemma simply apply Theorem 3.6 of [13] to the induced $G$-equivariant $\sigma$-homomorphism $M A\left(U_{2} \times G / \sim_{2}\right) \rightarrow M A\left(U_{1} \times G / \sim_{1}\right)$.

LEMMA 2.4. If $\delta\left(r_{1}(f)\right) \pi_{2} \circ \kappa(f)=\pi_{1}(f) \delta\left(d_{1}(f)\right)$ also holds on some i.c. of $V_{1}$, then $M\left(\kappa_{\theta}\right)$ and $M\left(\kappa_{\delta}\right)$ belong to the same morphism class.

Proof. By passing to an i.c. assume that both $\theta$ and $\delta$ provide a strict similarity between $\pi_{2} \circ \kappa$ and $\pi_{1}$. Now note that

$$
(\bar{\kappa}(u), x \theta(u)) x \theta(u) \delta(u)^{-1} x^{-1}=\left(\bar{\kappa}(u), x \delta(u) \theta(u)^{-1} x^{-1} x \theta(u)\right)=(\bar{\kappa}(u), x \delta(u)) .
$$

The Borel map $(u, x) \rightarrow x \theta(u) \delta(u)^{-1} x^{-1}$ is constant on $\sim_{1}$ equivalence classes because $\left(r_{1}(f), x\right) \rightarrow x \theta\left(r_{1}(f)\right) \delta\left(r_{1}(f)\right)^{-1} x^{-1}$ while

$$
\begin{aligned}
\left(d_{1}(f), x \pi_{1}(f)\right) \rightarrow & x \pi_{1}(f) \theta\left(d_{1}(f)\right) \delta\left(d_{1}(f)\right)^{-1} \pi_{1}(f)^{-1} x^{-1} \\
& =x \theta\left(r_{1}(f)\right) \pi_{2} \circ \kappa(f) \pi_{2} \circ \kappa(f)^{-1} \delta\left(r_{1}(f)\right)^{-1} x^{-1} \\
& =x \theta\left(r_{1}(f)\right) \delta\left(r_{1}(f)\right)^{-1} x^{-1} .
\end{aligned}
$$

Furthermore

$$
(u, x) y=\left(u, y^{-1} x\right) \rightarrow y^{-1} x \theta(u) \delta(u)^{-1} x^{-1} y .
$$

Hence $(u, x) \rightarrow x \theta(u) \delta(u)^{-1} x^{-1}$ factors to give a Borel map $\beta: U_{1} \times G / \sim_{1} \rightarrow G$ which satisfies $\beta(s x)=x^{-1} \beta(s) x$.

Before dealing further with $\beta$, observe that Theorem 2.1 of [13] indicates the existence of a Borel map from $U_{1} \times G / \sim_{1}$ to $M\left(V_{1} \pi_{1}\right)$ which induces the $G$ equivariant $\sigma$-isomorphism between their respective measure algebras. This map can be regularized by the process of Theorem 3.6 of [13] to give a measurable (in this case necessarily Borel too) map $\iota_{1}: U_{1} \times G / \sim_{1} \rightarrow M\left(V_{1} \pi_{1}\right)$ which is $G$ equivariant on an invariant conull set. The same reasoning gives a map $\iota_{2}: U_{2} \times G / \sim_{2}$ $\rightarrow M\left(V_{2} \pi_{2}\right)$ with similar properties. 
Let $\eta_{\theta}: U_{1} \times G / \sim_{1} \rightarrow U_{2} \times G / \sim_{2}$ and $\eta_{\delta}: U_{1} \times G / \sim_{1} \rightarrow U_{2} \times G / \sim_{2}$ be the maps which result from factoring $(u, x) \rightarrow(\bar{\kappa}(u), x \theta(u))$ and $(u, x) \rightarrow(\bar{\kappa}(u), x \delta(u))$ respectively. Affixing a $*$ to a map to denote the induced $\sigma$-homomorphism, the following relations clearly hold: $M\left(\kappa_{\theta}\right)^{*}=\iota_{1}^{*-1} \circ \eta_{\theta}^{*} \circ \iota_{2}^{*}$ and $M\left(\kappa_{\delta}\right)^{*}=\iota_{1}^{*-1} \circ \eta_{\delta}^{*} \circ \iota_{2}^{*}$. If $G$ is given the $G$-space structure discussed in paragraph 2 of Theorem 1 and the measure class induced by $\beta$ from $U_{1} \times G / \sim_{1}$, then $\iota_{1}^{*-1} \circ \beta^{*}$ can be used to define a Borel map $\alpha: M\left(V_{1} \pi_{1}\right) \rightarrow G$ which satisfies $\alpha(t x)=x^{-1} \alpha(t) x$ on some invariant analytic conull set. This is again a consequence of Theorem 3.6 of [13]. By definition $\alpha^{*}=\iota_{1}^{*-1} \circ \beta^{*}$.

Putting the above facts together, there is some common invariant conull set where $M\left(\kappa_{\theta}\right) \circ \iota_{1}=\iota_{2} \circ \eta_{\theta}, M\left(\kappa_{\delta}\right) \circ \iota_{1}=\iota_{2} \circ \eta_{\delta}, \alpha \circ \iota_{1}=\beta$, and the following computations work: $M\left(\kappa_{\theta}\right) \circ \iota_{1}(s) \alpha \circ \iota_{1}(s)=M\left(\kappa_{\theta}\right) \circ \iota_{1}(s) \beta(s)=M\left(\kappa_{\theta}\right) \circ \iota_{1}(s \beta(s))=\iota_{2} \circ \eta_{\theta}(s \beta(s))$ $=\iota_{2}\left(\eta_{\theta}(s) \beta(s)\right)=\iota_{2} \circ \eta_{\delta}(s)=M\left(\kappa_{\delta}\right) \circ \iota_{1}(s)$. Hence $M\left(\kappa_{\theta}\right) \cdot \alpha=M\left(\kappa_{\delta}\right)$ almost everywhere as contended.

LEMMA 2.5. If $\psi$ is a homomorphism similar to $\kappa$, then $M\left(\psi_{\varepsilon}\right)$ is equivalent to $M\left(\kappa_{\theta}\right)$ for every choice of $\varepsilon$.

Proof. Suppose without loss of generality that $\delta\left(r_{1}(f)\right) \kappa(f)=\psi(f) \delta\left(d_{1}(f)\right)$ is a strict similarity between the strict homomorphisms $\kappa$ and $\psi$. Since $\theta\left(r_{1}(f)\right) \pi_{2} \circ \kappa(f)$ $=\pi_{1}(f) \theta\left(d_{1}(f)\right)$ and $\pi_{2}$ is taken strict, it follows that $\theta\left(r_{1}(f)\right) \pi_{2}\left(\delta\left(r_{1}(f)\right)\right)^{-1} \cdot \pi_{2} \circ \psi(f)$ $=\pi_{1}(f) \theta\left(d_{1}(f)\right) \pi_{2}\left(\delta\left(d_{1}(f)\right)\right)^{-1}$. Because

$$
\begin{aligned}
\left(\bar{\psi}(u), x \theta(u) \pi_{2} \circ \delta(u)^{-1}\right) \\
=\left(r_{2}(\delta(u)), x \theta(u) \pi_{2} \circ \delta(u)^{-1}\right) \sim_{2}\left(d_{2}(\delta(u)), x \theta(u) \pi_{2} \circ \delta(u)^{-1} \pi_{2} \circ \delta(u)\right) \\
=(\bar{\kappa}(u), x \theta(u)),
\end{aligned}
$$

the two maps $(u, x) \rightarrow\left(\bar{\psi}(u), x \theta(u) \pi_{2} \circ \delta(u)^{-1}\right)$ and $(u, x) \rightarrow(\bar{\kappa}(u), x \theta(u))$ factor to give the same map $U_{1} \times G / \sim_{1} \rightarrow U_{1} \times G / \sim_{2}$. Finish the proof by applying the result of the last lemma.

REMARK. Let $\delta: T_{1} \rightarrow T_{2}$ be a map in some morphism class of $\mathrm{R}(G)$. Then $M\left(R(\delta)_{\theta}\right)=\delta$ if $\theta$ equals the identity of $G$ everywhere. Hence $M(R([\delta]))=[\delta]$ too.

PROPOSITION 2.6. If $[\kappa]: V_{1} \pi_{1} \rightarrow V_{2} \pi_{2}$ and $[\psi]: V_{2} \pi_{2} \rightarrow V_{3} \pi_{3}$ are $\mathrm{M}(G)$ morphisms, then $M([\psi] \circ[\kappa])=M([\psi]) \circ M([\kappa])$.

Proof. Assume that $\psi$ and $\kappa$ are composable and that all homomorphisms and similarities are strict. From the two relations $\theta_{2}\left(r_{2}(h)\right) \pi_{3} \circ \psi(h)=\pi_{2}(h) \theta_{2}\left(d_{2}(h)\right)$ and $\theta_{1}\left(r_{1}(f)\right) \pi_{2} \circ \kappa(f)=\pi_{1}(f) \theta_{1}\left(d_{1}(f)\right)$ follows the relation $\theta_{1}\left(r_{1}(f)\right) \theta_{2} \circ \bar{\kappa}\left(r_{1}(f)\right) \pi_{3} \circ \psi$ $\circ \kappa(f)=\pi_{1}(f) \theta_{1}\left(d_{1}(f)\right) \theta_{2} \circ \bar{k}\left(d_{1}(f)\right)$. Observe that the Borel map

$$
(u, x) \rightarrow\left(\bar{\psi} \circ \bar{\kappa}(u), x \theta_{1}(u) \theta_{2} \circ \bar{\kappa}(u)\right)
$$

from $U_{1} \times G$ to $U_{3} \times G$ is the composition of the two Borel maps $(u, x)$ $\rightarrow\left(\bar{\kappa}(u), x \theta_{1}(u)\right)$ and $(w, y) \rightarrow\left(\bar{\psi}(w), y \theta_{2}(w)\right)$ from $U_{1} \times G$ to $U_{2} \times G$ and $U_{2} \times G$ to $U_{3} \times G$ respectively. The same statement can be made for the corresponding 
factored maps. This behavior is transferred to the induced $\sigma$-homomorphisms and from there to the resulting $\mathrm{R}(G)$ homomorphisms.

Proposition 2.7. Given the morphism $[\kappa]: V \pi \rightarrow R(T)$ in $\mathrm{M}(G)$, where $T$ is a $G$-space in $\mathrm{R}(G)$, there is an $\mathrm{R}(G)$ morphism $[\kappa]^{\wedge}: M(V \pi) \rightarrow T$.

Proof. Just put $[\kappa]^{\wedge}=M([\kappa])$.

Proposition 2.8. Given an $\mathrm{R}(G)$ morphism $[\psi]: M(V \pi) \rightarrow T$, where $V \pi$ is a virtual group over $G$, there is an $\mathrm{M}(G)$ morphism $[\psi]^{\#}: V \pi \rightarrow R(T)$.

Proof. Let us first observe that there is a map $\varepsilon: U \times G \rightarrow M(V \pi)$ belonging to some $\mathrm{R}(G)$ morphism class such that the induced $\sigma$-homomorphism $\varepsilon^{*}$ gives the natural inclusion of the measure algebra over $M(V \pi)$ into the measure algebra over $U \times G$. As Theorem 7.8 of [13] shows, $f \rightarrow \pi^{\prime}(f)=(\varepsilon(r(f), e), \pi(f))$ is a $\mathrm{M}(G)$ homomorphism from $V \pi$ to $R(M(V \pi))$, where $e$ is the identity of $G$. Now set [ $\psi]^{\#}$ $=R([\psi]) \circ\left[\pi^{\prime}\right]$.

LEMma 2.9. $M\left(\left[\pi^{\prime}\right]\right)=\left[\iota_{d}\right]$, where $\pi^{\prime}$ is the $\mathrm{M}(G)$ homomorphism of the last proposition and $\iota_{d}$ is the identity map $M(V \pi) \rightarrow M(V \pi)$.

Proof. Let $\rho: R(M(V \pi)) \rightarrow T$ be projection onto $G$. Then $\rho \circ \pi^{\prime}=\pi$, so put $\theta=e$ and calculate $M\left(\pi_{\theta}^{\prime}\right) .(u, x) \rightarrow\left(\bar{\pi}^{\prime}(u), x\right)=((\varepsilon(u, e), e), x)$, which goes to $\varepsilon(u, e) x^{-1}$ $=\varepsilon(u, x)$ in the quotient map of $M(V \pi) \times G$ onto $M(V \pi)$.

Proposition 2.10. There is a one-to-one correspondence between the morphisms $[\kappa]: V \pi \rightarrow R(T)$ and $[\psi]: M(V \pi) \rightarrow T$. In fact,$\left([\kappa]^{\wedge}\right)^{\#}=[\kappa]$ and $\left([\psi]^{\#}\right)^{\wedge}=[\psi]$.

Proof. $\left([\psi]^{\#}\right)^{\wedge}=M\left(R([\psi]) \circ\left[\pi^{\prime}\right]\right)=M(R([\psi])) \circ M\left(\left[\pi^{\prime}\right]\right)=[\psi] \circ\left[\iota_{d}\right]=[\psi]$ by the last lemma and the remark after Lemma 2.5.

To prove that $\left([\kappa]^{\wedge}\right)^{\#}=[\kappa]$ suppose $\theta(r(f)) \rho \circ \kappa(f)=\pi(f) \theta(d(f))$ is a strict similarity, with $\rho: R(T) \rightarrow G$ projection onto $G . M\left(\kappa_{\theta}\right)$ is in the morphism class $[\kappa]^{\wedge}$ and is induced by factoring the map $(u, x) \rightarrow \bar{\kappa}(u) \theta(u)^{-1} x^{-1}$ of $U \times G$ into $T$. Indeed, if $\varepsilon: U \times G \rightarrow M(V \pi)$ is the map described in Proposition 2.8, then $M\left(\kappa_{\theta}\right) \circ \varepsilon$ and $(u, x) \rightarrow \bar{\kappa}(u) \theta(u)^{-1} x^{-1}$ must agree on some conull invariant analytic set of $U \times G$. Also note that $\bar{\kappa}(r(f)) \rho \circ \kappa(f)=d(\bar{\kappa}(r(f)), \rho \circ \kappa(f))=d(\kappa(f))=\bar{\kappa}(d(f))$. Hence on some i.c. of $V$,

$$
\begin{aligned}
R\left(M\left(\kappa_{\theta}\right)\right) & \circ \pi^{\prime}(f) \\
& =\left(M\left(\kappa_{\theta}\right)(\varepsilon(r(f), e)), \pi(f)\right)=\left(\bar{\kappa}(r(f)) \theta(r(f))^{-1}, \pi(f)\right) \\
& =\left(\bar{\kappa}(r(f)) \theta(r(f))^{-1}, \theta(r(f)) \rho \circ \kappa(f) \theta(d(f))^{-1}\right) \\
& =\left(\bar{\kappa}(r(f)) \theta(r(f))^{-1}, \theta(r(f))\right)(\bar{\kappa}(r(f)), \rho \circ \kappa(f))\left(\bar{\kappa}(r(f)) \rho \circ \kappa(f), \theta(d(f))^{-1}\right) \\
& =\left(\bar{\kappa}(r(f)), \theta(r(f))^{-1}\right)^{-1} \kappa(f)\left(\bar{\kappa}(d(f)), \theta(d(f))^{-1}\right) .
\end{aligned}
$$

Thus $u \rightarrow\left(\bar{\kappa}(u), \theta(u)^{-1}\right)$ implements a similarity between $R\left(M\left(\kappa_{\theta}\right)\right) \circ \pi^{\prime}$ and $\kappa$.

Proposition 2.11 (Naturality).

(a) Given morphisms [ $[\kappa]: V_{1} \pi_{1} \rightarrow V_{2} \pi_{2}$ and [ $[\psi]: V_{2} \pi_{2} \rightarrow R(T)$ in $\mathrm{M}(G)$, $\left([\psi]^{\wedge} \circ M([\kappa])\right)^{\#}=[\psi] \circ[\kappa]$. 
(b) Given morphisms $[\kappa]: T_{1} \rightarrow T_{2}$ and $[\psi]: M(V \pi) \rightarrow T_{1}$ in $\mathrm{R}(G), R([\kappa]) \circ[\psi]^{\#}$ $=([\kappa] \circ[\psi])^{\#}$.

Proof. (a) $\left.\left([\psi]^{\wedge} \circ M([\kappa])\right)\right)^{\#}=R(M([\psi]) \circ M([\kappa])) \circ\left[\pi^{\prime}\right]=R(M([\psi] \circ[\kappa])) \circ\left[\pi^{\prime}\right]$ $=[\psi] \circ[\kappa]$.

(b) $R([\kappa]) \circ[\psi]^{\#}=R([\kappa]) \circ R([\psi]) \circ\left[\pi^{\prime}\right]=R([\kappa] \circ[\psi]) \circ\left[\pi^{\prime}\right]=([\kappa] \circ[\psi]) \#$.

\section{Applications of the Adjoint Functor Theorem.}

Discrete flow under a function. Consider the virtual group $S \times Z$ formed by an ergodic action of the integers on an analytic Borel space $S$. Given a Borel function $f: S \rightarrow Z$ with positive integer values let us define a virtual group homomorphism $\pi: S \times Z \rightarrow Z$ as follows:

$$
\begin{aligned}
\pi(s, 0) & =0, \\
\pi(s, m) & =-\sum_{l=0}^{m-1} f\left(s^{l}\right), \quad m>0, \\
\pi(s,-m) & =\sum_{l=1}^{m} f\left(s^{-l}\right),
\end{aligned}
$$

where $s^{l}$ indicates the action of the integer $l$ on the point $s$. To clarify matters somewhat note that the homomorphism $\pi$ is uniquely determined by the Borel function $s \rightarrow \pi(s, 1)=-f(s)$. Indeed $\pi(s, n)=\pi(s, n-1)+\pi\left(s^{n-1}, 1\right)$ and $\pi(s,-n)+\pi\left(s^{-n}, n\right)$ $=\pi(s, 0)=0$.

Now look at the Borel subspace $E=\{(s, n): 0 \leqq n<f(s)\}$ of $S \times \boldsymbol{Z}$ with measure class the product measure class restricted to $E$. Suppose it can be shown that $E$ meets each $\sim$ equivalence class in exactly one point. Then it will follow that the natural map $S \times Z / \sim \rightarrow E$ is a Borel isomorphism. (See Theorem 15, Corollary 2 and Proposition 2.4 of Chapter 1 of [9].)

So assume $s \in S$ and $n \geqq 0$. Then there is a unique integer $m$ with $f\left(s^{m}\right)>n+\pi(s, m)$ $\geqq 0$. For either $f(s)>n \geqq 0$ or $n \geqq f\left(s^{m-1}\right)+\cdots+f(s)$ for some largest integer $m>0$. To show that $m$ is unique suppose $k>m$. Then

$$
\begin{aligned}
n+\pi(s, k) & =n+\pi(s, m)+\sum_{l=m}^{k-1} \pi\left(s^{l}, 1\right) \\
& =n+\pi(s, m)-f\left(s^{m}\right)-\sum_{l=m+1}^{k-1} f\left(s^{l}\right)<0 .
\end{aligned}
$$

This reduces the problem to eliminating the possibility $k<m$. The case $k<0$ is easily disposed of since $f\left(s^{k}\right) \leqq \pi(s, k)$. Thus assume $0 \leqq k<m$. Then $f\left(s^{k}\right)$ $>n+\pi(s, k) \geqq 0$ implies $f\left(s^{k}\right)+f\left(s^{k-1}\right)+\cdots+f(s)>n$, contradicting the choice of $m$.

In case $n<0$ the above assertion is also true. Since $\pi(s, m) \leqq 0$ for $m \geqq 0$, clearly no $m \geqq 0$ satisfies

$$
f\left(s^{m}\right)>n+\pi(s, m) \geqq 0 .
$$

Thus consider the largest $m$ with $-n>f\left(s^{-m+1}\right)+\cdots+f\left(s^{-1}\right)$. It is easy to verify 
that $f\left(s^{-m}\right)>n+\pi(s,-m) \geqq 0$. Suppose $k>m$. Then $-n \leqq f\left(s^{-k+1}\right)+\cdots+f\left(s^{-1}\right)$, so

$$
f\left(s^{-k}\right) \leqq n+\sum_{l=1}^{k} f\left(s^{-l}\right)=n+\pi(s,-k) .
$$

On the other hand, if $0 \leqq k<m,-n>f\left(s^{-k}\right)+\cdots+f\left(s^{-1}\right)=\pi(s,-k)$ so $0>n$ $+\pi(s,-k)$.

Next define a $Z$ action on $E$ by $(s, n) l=\left(s^{m}, n+l+\pi(s, m)\right)$, where $0 \leqq n+l+\pi(s, m)$ $<f\left(s^{m}\right)$. Because $Z$ is commutative, the $Z$ action on $S \times Z$ as described in Proposition 2.1 can be taken to be $(s, n) l=(s, n+l)$. Under these definitions the natural map $S \times Z / \sim \rightarrow E$ is obviously $Z$-equivariant. To complete the identification of $S \times Z / \sim$ with $E$ suppose $N$ is a null set in $E$. Then $(s, n) \in S \times Z$ maps into $N$ iff $\left(s^{m}, n+\pi(s, m)\right) \in N$. Hence

$$
s \in \bigcup_{k=0}^{\infty} \bigcup_{m=-\infty}^{\infty}\left\{t:\left(t^{m}, k\right) \in N\right\}
$$

which is a null set by Fubini's Theorem. Since this is true independently of $n$, the inverse image of $N$ is null. It also follows immediately that the image of a null set is null.

Kakutani's induced transformation. Again suppose the integers define an ergodic action on an analytic Borel space $S$. Let $A$ be any Borel set in $S$ of positive measure. If the given measure class on $S$ possesses a finite invariant measure or if $S$ is nonatomic, then with the exception of a null set every point of $A$ enters $A$ infinitely often. (See Theorem 1.15 of [1].) Define a virtual group $V^{A}$ to consist of all pairs $(s, n)$ from $A \times Z$ with $s \in A$ and $s^{n} \in A$. It is easy to see that $V^{A}$ is a groupoid. Furthermore, restricting the measure class of $V$ to the contraction $V^{A}$ gives a virtual group which is similar to $V$. (See Theorem 6.18 of [13].)

Assuming that almost every point of $A$ enters $A$ infinitely often, we define a virtual group homomorphism $\pi: V^{A} \rightarrow Z$. First some notation. If $s \in A$ and $s$ enters $A$ again, let $p(s)$ be the first positive integer with $s^{p(s)} \in A$. Similarly, let $n(s)$ be the first negative integer with $s^{n(s)} \in A$, provided such an integer exists. It suffices to define $\pi$ on an i.c. of $V^{A}$. Now according to Kakutani's classic argument, there is a Borel set $B \subset A$ differing from $A$ by a null set such that the map $s \rightarrow s^{p(s)}=T_{B}(s)$ is defined and is a Borel isomorphism of $B$ onto itself. Furthermore, both the image and inverse image of every null set is null under $T_{B}$. (See p. 193, Volume 1 of [3].) $\pi$ is defined on the i.c. $V^{A} \mid B$ as follows:

$$
\begin{aligned}
& \pi(s, 0)=0 \\
& \pi(s, m)=-k \text {, where } m>0, m=m_{1}+\cdots+m_{k} \\
& \text { and } m_{1}=p(s), m_{2}=p\left(s^{m_{1}}\right), \ldots, m_{k}=p\left(s^{m_{1}+\cdots+m_{k-1}}\right) \text {, } \\
& \pi(s, m)=k, \quad \text { where } m<0, m=m_{1}+\cdots+m_{k} \\
& \text { and } m_{1}=n(s), m_{2}=n\left(s^{m_{1}}\right), \ldots, m_{k}=n\left(s^{m_{1}+\cdots+m_{k-1}}\right) \text {. }
\end{aligned}
$$


It should be fairly clear that $\pi$ is a Borel map and algebraically a strict homomorphism. For instance,

$$
\begin{aligned}
& \{(s, m): \pi(s, m)=-k\} \\
& =\bigcup_{m=k}^{\infty}\left\{(s, m): s \in \bigcup_{\substack{m=m_{1}+\ldots+m_{k} \\
m_{1}>0}}\left(B \cap\left(t: \underset{\substack{l \neq m_{1}, m_{1}+m_{2}, \ldots, m \\
0 \leqq l \leqq m}}{t^{l}} \in B^{c}\right)\right.\right. \\
& \left.\left.\cap\left(t: \underset{l=m_{1}, m_{1}+m_{2}, \ldots, m}{t^{l}} \in B\right)\right)\right\} \text {. }
\end{aligned}
$$

The aim in defining $\pi:\left.V^{A}\right|_{B} \rightarrow Z$ is to show that the integer action defined by $T_{B}$ on $B$ can be identified with the integer action on $B \times Z / \sim$. With this in mind map any point $(s, n)$ of $B \times Z$ into $T_{B}^{n}(s)$. To prove that this map is constant on $\sim$ equivalence classes suppose $m=p(s)$. Then

$$
\left(s^{m}, n+\pi(s, m)\right) \rightarrow T_{B}^{n-1}\left(s^{m}\right)=T_{B}^{n}\left(T_{B}^{-1}\left(s^{m}\right)\right)=T_{B}^{n}(s) .
$$

Since $(s, n+m) \rightarrow T_{B}^{n+m}(s)=T_{B}^{n}\left(T_{B}^{m}(s)\right)$, the map is clearly $Z$-equivariant. If $T_{B}^{n}(s)$ $=T_{B}^{m}(t)$, then $T_{B}^{n-m}(s)=t$ and so $n-m=-\pi(s, k)$ for some integer $k$. But this entails $\left(s^{k}, n+\pi(s, k)\right)=(t, m)$ and so the map factors to give a bijection from $B \times Z / \sim$ onto $B$. By fixing $n$ and using the fact that $T_{B}$ is a Borel isomorphism, it is easy to verify that this bijection is a Borel isomorphism. In similar fashion one can show that the image and inverse image of a null set is null.

A virtual group homomorphism with a special property. We wish to construct a virtual group homomorphism with the property that the inverse image of some null set of units is not null under the unit map. This would indicate a real difference between the definition of homomorphism we have adopted and its replacement by a definition stipulating that the inverse image of every null set of units be null under the unit map. The latter definition has the advantage of eliminating composability problems between homomorphisms. However, it has the defect of not permitting one to pre- and post-multiply a homomorphism $\kappa$ by a compatible Borel function $\theta, \theta(r(f)) \kappa(f)(d(f))^{-1}$, and still wind up with a homomorphism.

To construct the desired homomorphism let $S$ be the unit circle with Lebesgue measure and suppose the integers act on $S$ by an irrational rotation. Define a virtual group homomorphism $\pi: S \times Z \rightarrow R$ into the reals by $\pi(s, n)=n$. Then the quotient space $S \times \boldsymbol{R} / \sim$ with the quotient Borel structure is Borel isomorphic and measure theoretically the same as $S \times[0,1)$ equipped with the obvious product measure. If $(s, t) x$ is defined to be $\left(s^{-[t-x]}, t-x-[t-x]\right)$, where $[t-x]$ is the unique integer $0 \leqq t-x-[t-x]<1$, then $S \times R / \sim$ and $S \times[0,1)$ are even Borel isomorphic as $\boldsymbol{R}$-spaces. (See the example at the end of Chapter 4 of [13].) Now consider the Borel map $s \rightarrow(s, 0)$ of $S$ into $S \times[0,1)$. It clearly satisfies $\left(s^{n}, 0\right)=(s, 0) n$. On the one hand, the inverse image of the Borel null set $S \times\{0\}$ is all of $S$. On the other hand, suppose $B$ is an $R$-invariant null set of $S \times[0,1)$. To show that the inverse image of $B$ under the map $s \rightarrow(s, 0)$ is null it suffices to prove that the section $B_{\{0\}}$ is null in $S$. But this follows from the fact that $B_{\{0\}} \times[0,1) \subset B$. Thus the Borel map $(s, n) \rightarrow$ 
$((s, 0), n)$ of $S \times \boldsymbol{Z}$ into $(S \times[0,1)) \times \boldsymbol{R}$ is a virtual group homomorphism with the property that the inverse image of some null sets of units is not null under the unit map.

More comments on the two definitions of virtual group homomorphism. This has little to do with the Adjoint Functor Theorem, but let us indicate a case where the definitions of virtual homomorphisms given in the last example coincide. Suppose $\kappa=R(\psi)$, where $\psi: T \rightarrow S$ belongs to an $\mathrm{R}(G)$ morphism class. $\psi$ can be identified with the unit map of $\kappa$. The saturated sets in $T$ and $S$ are simply the invariant sets. Next assume the measure classes of $T$ and $S$ both contain invariant probability measures. Let the given invariant probability measure on $S$ be $\nu$ and the invariant probability measure induced on $S$ by $\psi$ be $\mu$. Then $\mu$ is absolutely continuous with respect to $\nu$ on the $\sigma$-algebra of invariant Borel sets. We wish to show that $\mu$ is absolutely continuous with respect to $\nu$ on the $\sigma$-algebra of all Borel sets.

To do this we recall a result of Varadarajan. (See Theorem 4.1 of [16].) There is an operator $f \rightarrow \mathrm{U} f$ from the Banach space of all bounded real-valued Borel functions on $S$ into the Banach space of all bounded real-valued Borel functions on $S$ which are left invariant by the action of $G$. U is a conditional expectation operator for the $\sigma$-algebra of invariant Borel sets and every invariant probability measure. Now take a Borel set $A$ in $S$ with $\nu(A)=0$. Let $\chi_{A}$ be the characteristic function of $A$. Then $\chi_{A} \geqq 0 \nu$ and $\mu$-almost everywhere, $\operatorname{so} \nu(A)=\int \chi_{A} d \nu=\int\left(\mathrm{U} \chi_{A}\right) d \nu$ $=0$ and the absolute continuity of $\mu$ with respect to $\nu$ on the invariant Borel sets imply $\mu(A)=\int \chi_{A} d \mu=\int\left(\mathrm{U} \chi_{A}\right) d \mu=0$.

\section{Products of virtual groups.}

Theorem 3. Let $\left(V_{1}, C_{1}\right)$ and $\left(V_{2}, C_{2}\right)$ be analytic Borel groupoids. Then $\left(V_{1} \times V_{2}, C_{1} \times C_{2}\right)$ is an analytic Borel groupoid which is a virtual group if and only if both $\left(V_{1}, C_{1}\right)$ and $\left(V_{2}, C_{2}\right)$ are. $\left(V_{1} \times V_{2}, C_{1} \times C_{2}\right)$ is essentially transitive if and only if both $\left(V_{1}, C_{1}\right)$ and $\left(V_{2}, C_{2}\right)$ are. Similarly, $\left(V_{1} \times V_{2}, C_{1} \times C_{2}\right)$ is essentially principal if and only if both $\left(V_{1}, C_{1}\right)$ and $\left(V_{2}, C_{2}\right)$ are. If $\kappa_{1}: V_{1} \rightarrow W_{1}$ and $\kappa_{2}: V_{2} \rightarrow W_{2}$ are virtual group homomorphisms, then $\kappa_{1} \times \kappa_{2}: V_{1} \times V_{2} \rightarrow W_{1} \times W_{2}$ is a virtual group homomorphism. Furthermore, if $V_{1}$ and $V_{2}$ are similar to $W_{1}$ and $W_{2}$ respectively, then $V_{1} \times V_{2}$ is similar to $W_{1} \times W_{2}$.

Proof. Let $\left(V_{1}, C_{1}\right)$ and $\left(V_{2}, C_{2}\right)$ be analytic Borel groupoids. Consider the cartesian product $V_{1} \times V_{2}$. It is an analytic Borel space and has a natural groupoid structure. The composition $\left(f_{1}, f_{2}\right)\left(h_{1}, h_{2}\right)$ is defined to be $\left(f_{1} h_{1}, f_{2} h_{2}\right)$ whenever $d_{1}\left(f_{1}\right)=r_{1}\left(h_{1}\right)$ and $d_{2}\left(f_{2}\right)=r_{2}\left(h_{2}\right)$. Inversion is the Borel map $\left(f_{1}, f_{2}\right) \rightarrow\left(f_{1}, f_{2}\right)^{-1}$ $=\left(f_{1}^{-1}, f_{2}^{-1}\right)$. The right unit $d\left(f_{1}, f_{2}\right)$ of $\left(f_{1}, f_{2}\right)$ is $\left(d_{1}\left(f_{1}\right), d_{2}\left(f_{2}\right)\right)$ and the left unit $r\left(f_{1}, f_{2}\right)=\left(r_{1}\left(f_{1}\right), r_{2}\left(f_{2}\right)\right)$. Clearly both $d$ and $r$ are Borel maps. Their common range is $U_{1} \times U_{2}$, where $U_{i}$ is the set of units of $V_{i}, i=1$, 2. If $D_{i}$ is the domain of composition for $V_{i}$, then the domain of composition $D$ of $V_{1} \times V_{2}$ is the image of $D_{1} \times D_{2}$ under the natural Borel isomorphism of $\left(V_{1} \times V_{1}\right) \times\left(V_{2} \times V_{2}\right)$ onto $\left(V_{1} \times V_{2}\right) \times\left(V_{1} \times V_{2}\right)$. Hence $D$ is a Borel set. Composition is also a Borel map from $D$ to $V_{1} \times V_{2}$. 
Since the product measure class $C_{1} \times C_{2}$ on $V_{1} \times V_{2}$ is well defined, it is possible to choose symmetric probability measures $\mu_{i}$ from $C_{i}$ and consider only the product measure $\mu_{1} \times \mu_{2}$. (Symmetric means $\mu_{i}(A)=\mu_{i}\left(A^{-1}\right)$ for every Borel set $A$ in $V_{i}$.) Let us first prove that $\mu_{1} \times \mu_{2}$ is symmetric. Suppose $A_{1}$ and $A_{2}$ are Borel sets in $V_{1}$ and $V_{2}$ respectively. Then

$$
\begin{aligned}
\mu_{1} \times \mu_{2}\left(\left(A_{1} \times A_{2}\right)^{-1}\right) & =\mu_{1} \times \mu_{2}\left(A_{1}^{-1} \times A_{2}^{-1}\right)=\mu_{1}\left(A_{1}^{-1}\right) \mu_{2}\left(A_{2}^{-1}\right) \\
& =\mu_{1}\left(A_{1}\right) \mu_{2}\left(A_{2}\right)=\mu_{1} \times \mu_{2}\left(A_{1} \times A_{2}\right) .
\end{aligned}
$$

By finite additivity $\mu_{1} \times \mu_{2}\left(A^{-1}\right)=\mu_{1} \times \mu_{2}(A)$ whenever $A$ is a finite disjoint union of Borel rectangles. Passing to monotone limits, $\mu_{1} \times \mu_{2}\left(A^{-1}\right)$ and $\mu_{1} \times \mu_{2}(A)$ are seen to agree for all Borel sets $A$ in the product $\sigma$-algebra.

The same technique of verifying a certain property for Borel rectangles, then by additivity for finite disjoint unions of Borel rectangles, then for all Borel sets in the product $\sigma$-algebra by passing to monotone limits, can be used to establish the following. Let

$$
\mu_{1}=\int \mu_{1}^{u_{1}} d \bar{\mu}_{1}\left(u_{1}\right) \text { and } \mu_{2}=\int \mu_{2}^{u} d \bar{\mu}_{2}\left(u_{2}\right)
$$

be decompositions of $\mu_{1}$ and $\mu_{2}$ with respect to $r_{1}$ and $r_{2}$. Then

$$
\mu_{1} \times \mu_{2}=\int \mu_{1}^{u} \times \mu_{2}^{u} d \bar{\mu}_{1} \times \bar{\mu}_{2}\left(u_{1}, u_{2}\right)
$$

is the decomposition of $\mu_{1} \times \mu_{2}$ with respect to $r$. Now let us check the invariance of the measure class $C_{1} \times C_{2}$. Take conull Borel sets $U_{i}^{\prime} \subset U_{i}$ such that whenever $A_{i} \subset r_{i}^{-1}\left(d_{i}\left(f_{i}\right)\right)$ and $d_{i}\left(f_{i}\right), r_{i}\left(f_{i}\right) \in U_{i}^{\prime}, \mu_{i}^{r_{i}\left(f_{i}\right)}\left(f_{i} A_{i}\right)=0$ iff $\mu_{i}^{d_{i}\left(f_{i}\right)}\left(A_{i}\right)=0$. Assume that both $r\left(f_{1}, f_{2}\right)$ and $d\left(f_{1}, f_{2}\right)$ are in the conull Borel set $U_{1}^{\prime} \times U_{2}^{\prime}$. Then, for $A \subset r^{-1}\left(d\left(f_{1}, f_{2}\right)\right)$,

$$
\begin{aligned}
\mu_{1}^{r_{1}\left(f_{1}\right)} \times \mu_{2}^{r_{2}\left(f_{2}\right)}\left(\left(f_{1}, f_{2}\right) A\right) & \\
& =\int \mu_{1}^{r_{1}\left(f_{1}\right)}\left\{h_{1} \in r_{1}^{-1}\left(r_{1}\left(f_{1}\right)\right):\left(h_{1}, h_{2}\right) \in\left(f_{1}, f_{2}\right) A\right\} d \mu_{2}^{r_{2}\left(f_{2}\right)}\left(h_{2}\right) \\
& =\int \mu_{1}^{r_{1}\left(f_{1}\right)}\left\{h_{1} \in r_{1}^{-1}\left(r_{1}\left(f_{1}\right)\right): f_{1}^{-1} h_{1} \in A_{f_{2}^{-1} h_{2}}\right\} d \mu_{2}^{r_{2}\left(f_{2}\right)}\left(h_{2}\right) .
\end{aligned}
$$

By Fubini's Theorem $\mu_{1}^{r_{1}\left(f_{1}\right)} \times \mu_{2}^{r_{2}\left(f_{2}\right)}\left(\left(f_{1}, f_{2}\right) A\right)=0$ iff for $\mu_{2}^{r_{2}\left(f_{2}\right)}$ almost all $h_{2} \in r_{2}^{-1}\left(r_{2}\left(f_{2}\right)\right)$,

$$
\mu_{1}^{r_{1}\left(f_{1}\right)}\left\{h_{1} \in r_{1}^{-1}\left(r_{1}\left(f_{1}\right)\right): f_{1}^{-1} h_{1} \in A_{f_{2}^{-1} h_{2}}\right\}=0
$$

iff for $\mu_{2^{2}}^{r}\left(_{2}\right)$ almost all $h_{2} \in r_{2}^{-1}\left(r_{2}\left(f_{2}\right)\right)$,

$$
\mu_{1}^{d_{1}\left(f_{1}\right)}\left\{g_{1} \in r_{1}^{-1}\left(d_{1}\left(f_{1}\right)\right): g_{1} \in A_{f_{2}^{-1} h_{2}}\right\}=0
$$

iff for $\mu_{2}^{d_{2}\left(f_{2}\right)}$ almost all $g_{2} \in r_{2}^{-1}\left(d_{2}\left(f_{2}\right)\right)$,

$$
\mu_{1}^{d_{1}\left(f_{1}\right)}\left\{g_{1} \in r_{1}^{-1}\left(d_{1}\left(f_{1}\right)\right): g_{1} \in A_{g_{2}}\right\}=0
$$


iff $\mu_{1}^{d_{1}\left(f_{1}\right)} \times \mu_{2}^{d_{2}\left(f_{2}\right)}(A)=0$. This completes the proof that $\left(V_{1} \times V_{2}, C_{1} \times C_{2}\right)$ is an analytic Borel groupoid.

Next suppose $\left(V_{1}, C_{1}\right)$ and $\left(V_{2}, C_{2}\right)$ are virtual groups. To prove that $\left(V_{1} \times V_{2}, C_{1} \times C_{2}\right)$ is a virtual group it is enough to show that every saturated analytic set of units is null or conull. (See Theorem 4.2 of [13].) So let $A$ be a saturated analytic set of units in $U_{1} \times U_{2}$. The section $A_{u_{2}}=\left\{u_{1}:\left(u_{1}, u_{2}\right) \in A\right\}$ is saturated. It is analytic because it is the inverse image of $A$ under the Borel map $u_{1} \rightarrow\left(u_{1}, u_{2}\right)$. Hence the definition of ergodicity implies $A_{u_{2}}$ is either null or conull. Now the set $\left\{u_{2}: A_{u_{2}}\right.$ is null $\}$ is also saturated. It is measurable since $u_{2} \rightarrow \bar{\mu}_{1}\left(A_{u_{2}}\right)$ is measurable. Hence it too is null or conull. These dichotomies and Fubini's Theorem imply $A$ is null or conull.

On the other hand suppose either $\left(V_{1}, C_{1}\right)$ or $\left(V_{2}, C_{2}\right)$ fails to be a virtual group. Then one of them, say $\left(V_{1}, C_{1}\right)$, has a saturated analytic set of units $U_{1}^{\prime}$ which is neither null nor conull. But then $U_{1}^{\prime} \times U_{2}$ is a saturated analytic set of units in $U_{1} \times U_{2}$ which is neither null nor conull.

To show that $\left(V_{1} \times V_{2}, C_{1} \times C_{2}\right)$ is essentially transitive if and only if both $\left(V_{1}, C_{1}\right)$ and $\left(V_{2}, C_{2}\right)$ are, consider the saturation $r\left(d^{-1}\left(u_{1}, u_{2}\right)\right)$ of any unit $\left(u_{1}, u_{2}\right)$. $r\left(d^{-1}\left(u_{1}, u_{2}\right)\right)=r\left(d_{1}^{-1}\left(u_{1}\right) \times d_{2}^{-1}\left(u_{2}\right)\right)=r_{1}\left(d_{1}^{-1}\left(u_{1}\right)\right) \times r_{2}\left(d_{2}^{-1}\left(u_{2}\right)\right)$ is conull if and only if both $r_{1}\left(d_{1}^{-1}\left(u_{1}\right)\right)$ and $r_{2}\left(d_{2}^{-1}\left(u_{2}\right)\right)$ are conull.

For a virtual group $V$ let $V u=\{f \in V: d(f)=r(f)=u\}$ for every unit $u$ in $V$. To prove that $V_{1} \times V_{2}$ is essentially principal if and only if both $V_{1}$ and $V_{2}$ are, note that $V_{1} \times V_{2}\left(u_{1}, u_{2}\right)=V_{1} u_{1} \times V_{2} u_{2}$ for each pair $\left(u_{1}, u_{2}\right)$ in $U_{1} \times U_{2}$. Then

$$
\begin{aligned}
\left\{\left(u_{1}, u_{2}\right) \in U_{1} \times U_{2}:\right. & \left.V_{1} \times V_{2}\left(u_{1}, u_{2}\right)=\left\{\left(u_{1}, u_{2}\right)\right\}\right\} \\
& =\left\{u_{1} \in U_{1}: V_{1} u_{1}=\left\{u_{1}\right\}\right\} \times\left\{u_{2} \in U_{2}: V_{2} u_{2}=\left\{u_{2}\right\}\right\} .
\end{aligned}
$$

It follows from Fubini's Theorem that $\left\{\left(u_{1}, u_{2} \in U_{1} \times U_{2}: V_{1} \times V_{2}\left(u_{1}, u_{2}\right)\right.\right.$ $\left.=\left\{\left(u_{1}, u_{2}\right)\right\}\right\}$ is conull in $U_{1} \times U_{2}$ if and only if both $V_{1}$ and $V_{2}$ are essentially principal.

Next assume $\kappa_{1}: V_{1} \rightarrow W_{1}$ and $\kappa_{2}: V_{2} \rightarrow W_{2}$ are virtual group homomorphisms. Then $\kappa_{1} \times \kappa_{2}: V_{1} \times V_{2} \rightarrow W_{1} \times W_{2}$ is a Borel map and algebraically a homomorphism on some i.c. of $V_{1} \times V_{2}$. If $\bar{\kappa}_{i}$ is the unit map corresponding to $\kappa_{i}, i=1,2$, then $\bar{\kappa}_{1} \times \bar{\kappa}_{2}$ is the unit map corresponding to $\kappa_{1} \times \kappa_{2}$. Let $A$ be a saturated null set of units in $W_{1} \times W_{2}$. Then $\left\{u_{1}: \bar{\kappa}_{1}\left(u_{1}\right) \in A_{\bar{\kappa}_{2}\left(u_{2}\right)}\right\}$ is null whenever $A_{\bar{\kappa}_{2}\left(u_{2}\right)}$ is null since $A_{\bar{\kappa}_{2}\left(u_{2}\right)}$ is saturated and $\kappa_{1}$ is a homomorphism. Now consider the saturated set $\left\{w\right.$ a unit in $W_{2}: A_{w}$ is null $\}$. Because it is also conull, $\left\{u_{2}: A_{\bar{\kappa}_{2}\left(u_{2}\right)}\right.$ is null $\}$ is conull. Thus Fubini's Theorem implies that $\bar{\kappa}_{1} \times \bar{\kappa}_{2}^{-1}(A)$ is null.

Finally, given the similarity of $V_{i}$ and $W_{i}, i=1,2$, the similarity of $V_{1} \times V_{2}$ and $W_{1} \times W_{2}$ can be established by the following observation: If $\theta_{i}$ implements a similarity between the virtual group homomorphisms $\kappa_{i}$ and $\psi_{i}$, then $\theta_{1} \times \theta_{2}$ implements a similarity between $\kappa_{1} \times \kappa_{2}$ and $\psi_{1} \times \psi_{2}$.

EXAMPLE. Let $V=S \times Z$, where $S$ is the unit circle with Lebesgue measure and $Z$ acts on $S$ by an irrational rotation. For the virtual group $V \times V$ together with 
projection onto each coordinate to be the categorical product of $V$ with itself, it seems reasonable that the map id $\times$ id: $V \rightarrow V \times V$ in the diagram below should be a homomorphism. id $\times \mathrm{id}(f)=(f, f)$. But this is false by the following reasoning. Consider the saturation $\bigcup_{n, m \in Z} D \cdot(n, m)$ of the diagonal $D$ in the product space $S \times S$. Since the action of $Z \times Z$ on $S \times S$ leaves the measure invariant, $\bigcup_{n, m \in Z} D \cdot(n, m)$ is a saturated null set in $S \times S$. However, its inverse image under the unit map id ${ }^{-} \times \mathrm{id}^{-}$is all of $S$.

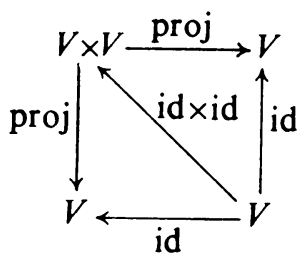

REMARKS. An analogue of Theorem 3 for countable products of virtual groups fails because Kakutani's Theorem for the absolute continuity of infinite product measures makes it impossible to define a single measure class on the countable product space. (See Theorem 22.36 of [2].) For the same reason trouble arises in verifying the invariance of the fiber measures for countable products.

Coproducts probably do not exist in the category of virtual groups since they would correspond to disjoint unions, which would destroy ergodicity.

The second theorem of this section provides a link between the Mackey functor and products of virtual groups.

THEOREM 4. Suppose $\pi_{1}: V_{1} \rightarrow G_{1}$ and $\pi_{2}: V_{2} \rightarrow G_{2}$ are virtual groups over the separable locally compact groups $G_{1}$ and $G_{2}$ respectively. Let $M_{i}$ be the Mackey Functor from $\mathrm{M}\left(G_{i}\right)$ to $\mathrm{R}\left(G_{i}\right), i=1$ or 2 . Similarly, let $M_{1,2}$ be the Mackey Functor from $\mathrm{M}\left(G_{1} \times G_{2}\right)$ to $\mathrm{R}\left(G_{1} \times G_{2}\right)$. Then $M_{1,2}\left(V_{1} \times V_{2} \pi_{1} \times \pi_{2}\right)$ is isomorphic as a standard Borel $G_{1} \times G_{2}$-space with invariant measure class to $M_{1}\left(V_{1} \pi_{1}\right) \times M_{2}\left(V_{2} \pi_{2}\right)$.

Proof. In more or less obvious notation define the equivalence relation $\sim$ on $U_{1} \times U_{2} \times G_{1} \times G_{2}$ by $\left(r_{1}\left(f_{1}\right), r_{2}\left(f_{2}\right), x_{1}, x_{2}\right) \sim\left(d_{1}\left(f_{1}\right), d_{2}\left(f_{2}\right), x_{1} \pi_{1}\left(f_{1}\right), x_{2} \pi_{2}\left(f_{2}\right)\right)$. Then we must show that $U_{1} \times U_{2} \times G_{1} \times G_{2} / \sim$ and $U_{1} \times G_{1} / \sim_{1} \times U_{2} \times G_{2} / \sim_{2}$ have measure algebras which are isomorphic as standard Boolean $G_{1} \times G_{2}$-spaces. This suffices since $U_{1} \times G_{1} / \sim_{1} \times U_{2} \times G_{2} / \sim_{2}$ and $M_{1}\left(V_{1} \pi_{1}\right) \times M_{2}\left(V_{2} \pi_{2}\right)$ do.

Now the trivial rearrangement $U_{1} \times G_{1} \times U_{2} \times G_{2}$ and redefinition of $\sim$ allow us to consider $\sim$ to be the product of $\sim_{1}$ and $\sim_{2}$. Noting that the canonical bijection $U_{1} \times G_{1} \times U_{2} \times G_{2} / \sim \rightarrow U_{1} \times G_{1} / \sim_{1} \times U_{2} \times G_{2} / \sim_{2}$ is Borel and $G_{1} \times G_{2^{-}}$ equivariant, it is enough to prove that the $\sigma$-homomorphism it induces is actually a $\sigma$-isomorphism. Under this map the inverse image of a set is null iff the set itself is null. Hence the $\sigma$-homomorphism is one-to-one. To prove that it is onto write $U_{1} \times G_{1}=S_{1}$ and $U_{2} \times G_{2}=S_{2}$ and let $\mu_{1}$ and $\mu_{2}$ be probability measures in the measure classes of $S_{1}$ and $S_{2}$ respectively. Give $S_{1} \times S_{2}$ the product probability 
measure $\mu_{1} \times \mu_{2}$. Since the $\sigma$-homomorphism induced from $S_{1} \times S_{2} / \sim \rightarrow S_{1} / \sim_{1}$ $\times S_{2} / \sim_{2}$ is measure preserving and every measure algebra is complete as a metric space, the problem reduces to showing that every measurable set $A$ in $S_{1} \times S_{2}$ which is saturated with respect to $\sim$ can be approximated by Borel sets in $S_{1} / \sim_{1}$ $\times S_{2} / \sim_{2}$.

The idea is to approximate $A$ by finite disjoint unions of measurable rectangles $\bigcup_{i=1}^{n} B_{i} \times C_{i}$, where each $B_{i}$ is saturated with respect to $\sim_{1}$ and each $C_{i}$ with respect to $\sim_{2}$. So first of all choose a sequence $\left\{C_{n}\right\}_{n=1}^{\infty}$ of measurable sets in $S_{2}$ which are saturated with respect to $\sim_{2}$ and which give a dense subset of the separable measure algebra over $S_{2} / \sim_{2}$. For $\varepsilon>0$ and $n$ a positive integer define $B_{n}=\left\{x_{1} \in S_{1}\right.$ : $\left.\mu_{2}\left(A^{x_{1}} \triangle C_{n}\right)<\varepsilon\right\}$. It follows immediately that each $B_{n}$ is measurable and saturated with respect to $\sim_{1}$. Also Fubini's Theorem implies that $\bigcup_{n=1}^{\infty} B_{n}$ differs from $S_{1}$ by a null set. Now put $D_{1}=B_{1}, D_{2}=B_{2}\left|B_{1}, D_{3}=B_{3}\right|\left(B_{1} \cup B_{2}\right)$, etc. The $D_{n}$ 's have the same properties as the $B_{n}$ 's with the additional property of disjointness. Let us see how closely $A$ is approximated by $\bigcup_{i=1}^{n} D_{i} \times C_{i}$ :

$$
\begin{aligned}
\iint \mid \chi_{A}-\chi_{D_{1} \times C_{1}}- & \cdots-\chi_{D_{n} \times C_{n}} \mid d \mu_{2} d \mu_{1} \\
\leqq & \int_{D_{1}} \int\left|\chi_{A}-\chi_{D_{1} \times C_{1}}\right| d \mu_{2} d \mu_{1}+\cdots \\
& \quad+\int_{D_{n}} \int\left|\chi_{A}-\chi_{D_{n} \times C_{n}}\right| d \mu_{2} d \mu_{1}+\int_{S_{1} \backslash \cup_{i=1}^{n} D_{i}} \int\left|\chi_{A}\right| d \mu_{2} d \mu_{1} \\
\leqq & \varepsilon \mu_{1}\left(\bigcup_{i=1}^{n} D_{i}\right)+\mu_{1}\left(S_{1} \backslash\left(\bigcup_{i=1}^{n} D_{i}\right)\right) .
\end{aligned}
$$

Hence for $n$ large enough $\bigcup_{i=1}^{n} D_{i} \times C_{i}$ approximates $A$ within $2 \varepsilon$ say. This estimate establishes the theorem.

REMARK. Suppose [ $\kappa$ ]: $W_{1} \rho_{1} \rightarrow V_{1} \pi_{1}$ is a morphism in $M\left(G_{1}\right)$ and [ $\left.\theta\right]: W_{2} \rho_{2}$ $\rightarrow V_{2} \pi_{2}$ a morphism in $\mathrm{M}\left(G_{2}\right)$. Then $M_{1}(\kappa) \times M_{2}(\theta)$ is essentially the same as $M_{1,2}(\kappa \times \theta)$. Since nothing novel is involved here, we leave the details to the interested reader.

\section{BIBLIOGRAPHY}

1. N. Friedman, Introduction to ergodic theory, Van Nostrand Reinhold, New York, 1970.

2. E. Hewitt and K. Stromberg, Real and abstract analysis. A modern treatment of the theory of functions of a real variable, Springer-Verlag, New York, 1965. MR 32 \#5826.

3. K. Jacobs, Lecture notes on ergodic theory, 1962/63. Parts I, II, Matematisk Institut, Aarhus Universitet, Aarhus, 1963, pp. 1-207, 208-505. MR 28 \#3138; errata, 28, 1247.

4. F. W. Lawvere, Equality in hyperdoctrines and comprehension schema as an adjoint functor, Proc. Sympos. Pure Math., vol. 17, Amer. Math. Soc., Providence, R. I., 1970, pp. 1-14. MR 41 \#1829.

5. G. W. Mackey, Ergodic theory, group theory, and differential geometry, Proc. Nat. Acad. Sci. U.S.A. 50 (1963), 1184-1191. MR 29 \#2325.

6. —_, Ergodic theory and virtual groups, Math. Ann. 166 (1966), 187-207. MR 34 \#1444. 
7. G. W. Mackey, Virtual groups, Topological Dynamics (Sympos. Colorado State Univ., Ft. Collins, Colo., 1967), Benjamin, New York, 1968, pp. 335-364. MR 39 \#2907.

8. - Induced representations of groups and quantum mechanics, Benjamin, New York, 1968.

9. C. C. Moore and L. Auslander, Unitary representations of solvable Lie groups, Mem. Amer. Math. Soc. No. 62 (1966), pp. 2-18. MR 34 \#7723.

10. C. C. Moore, On the Frobenius reciprocity theorem for locally compact groups, Pacific J. Math. 12 (1962), 359-365. MR 25 \#5134.

11. B. Pareigis, Categories and functors, Academic Press, New York, 1970.

12. K. R. Parthasarathy, Probability measures on metric spaces, Probability and Mathematical Statistics, no. 3, Academic Press, New York, 1967. MR 37 \#2271.

13. A. Ramsay, Virtual groups and group actions, Advances in Math. 6 (1971), 253-322.

14. M. A. Rieffel, Induced Banach representations of Banach algebras and locally compact groups, J. Functional Analysis 1 (1967), 443-491. MR 36 \#6544.

15. K. Lange, A. Ramsay and G.-C. Rota, Frobenius reciprocity in ergodic theory, Bull. Amer. Math. Soc. 77 (1971), 713-718.

16. V. S. Varadarajan, Groups of automorphisms of Borel spaces, Trans. Amer. Math. Soc. 109 (1963), 191-220. MR 28 \#3139.

17. - Geometry of quantum theory. Vol. II, Van Nostrand Reinhold, New York, 1970.

18. J. J. Westman, Cohomology for ergodic groupoids, Trans. Amer. Math. Soc. 146 (1969), 465-471. MR 41 \#431.

Department of Mathematics, University of New Hampshire, Durham, New HAMPSHIRE 03824 